PROCEEDINGS OF THE

AMERICAN MATHEMATICAL SOCIETY

Volume 126, Number 11, November 1998, Pages 3177-3181

S 0002-9939(98)04473-6

\title{
ON THE POINCARÉ SERIES AND CARDINALITIES OF FINITE REFLECTION GROUPS
}

\author{
JOHN R. STEMBRIDGE
}

(Communicated by Jeffry N. Kahn)

\begin{abstract}
Let $W$ be a crystallographic reflection group with length function $\ell(\cdot)$. We give a short and elementary derivation of the identity $\sum_{w \in W} q^{\ell(w)}=$ $\prod\left(1-q^{\mathrm{ht}(\alpha)+1}\right) /\left(1-q^{\mathrm{ht}(\alpha)}\right)$, where the product ranges over positive roots $\alpha$, and $\operatorname{ht}(\alpha)$ denotes the sum of the coordinates of $\alpha$ with respect to the simple roots. We also prove that in the noncrystallographic case, this identity is valid in the limit $q \rightarrow 1$; i.e., $|W|=\prod($ ht $(\alpha)+1) / \operatorname{ht}(\alpha)$.
\end{abstract}

\section{INTRODUCTION}

Let $W$ be a finite real reflection group of rank $n$ with the usual associated data: root system $\Phi \subset \mathbf{R}^{n}$, positive roots $\Phi^{+}$, simple roots $\alpha_{1}, \ldots, \alpha_{n}$, corresponding simple reflections $s_{1}, \ldots, s_{n} \in W$, and inner product $\langle$,$\rangle . For w \in W$, we let $\ell(w)$ denote the minimum length among all expressions $w=s_{i_{1}} \cdots s_{i_{l}}, \operatorname{sgn}(w)=$ $(-1)^{\ell(w)}=\operatorname{det}(w)$, and $\Phi(w)=\left\{\alpha \in \Phi^{+}: w \alpha \in-\Phi^{+}\right\}$. For roots $\alpha \in \Phi$, we let $\alpha^{\vee}$ denote the co-root $2 \alpha /\langle\alpha, \alpha\rangle$, and use the notation $\alpha>0$ to indicate $\alpha \in \Phi^{+}$. Recall that $\Phi$ is said to be crystallographic if $\left\langle\alpha, \beta^{\vee}\right\rangle \in \mathbf{Z}$ for all $\alpha, \beta \in \Phi$. Standard references are $[\mathrm{B}]$ and $[\mathrm{H}]$.

The purpose of this paper is two-fold. First, we give a very short and elementary proof of a product formula for the Poincare series $\sum_{w \in W} q^{\ell(w)}$ in the crystallographic case. Our proof is based on Macdonald's approach [M], in which the formula is deduced by specializing a more general identity in the group ring of the root lattice (Theorem 1). However the proof we give is, in our opinion, significantly simpler in the details. Indeed, it involves nothing more sophisticated than elementary manipulations and the fact that for all $w \in W, \ell(w)=|\Phi(w)|$. Eric Opdam [private communication] has pointed out that a similar proof appears in the unpublished Ph. D. thesis of René Beerends [Be]. Our proof is arguably more elementary than Beerends' in that the issue of divisibility of expressions by the Weyl denominator does not arise.

Our second objective is to show that, although this particular product formula for the Poincaré series is not valid in the non-crystallographic case, it is valid in the limit $q \rightarrow 1$, and this leads to a new formula for the cardinality of $W$ (Theorem 3). Of course, there is an explicit product formula for the Poincare series of any real reflection group due to Solomon (e.g., $[\mathrm{H}, \S 3.15]$ ), and the cardinality of any such

Received by the editors October 9, 1996 and, in revised form, March 29, 1997.

1991 Mathematics Subject Classification. Primary 20H15, 20F55.

The author was partially supported by a grant from the NSF. 
group is the product of the degrees of the fundamental invariants, but these facts require lengthier arguments.

\section{The PoInCARÉ SERIES}

In the following, it will be convenient to work in the group ring of $\mathbf{R}^{n}$, with the basis elements written as formal exponentials $e^{\lambda}$ for $\lambda \in \mathbf{R}^{n}$.

1.1. Define $\rho=\sum_{\alpha>0} \alpha / 2$. Since $s_{i}$ permutes $\Phi^{+}-\left\{\alpha_{i}\right\}$, it follows that $s_{j} \rho=$ $\rho-\alpha_{j}$. On the other hand, the reflection $s_{j}$ acts via $s_{j} \rho=\rho-\left\langle\rho, \alpha_{j}^{\vee}\right\rangle \alpha_{j}$, so we have

$$
\left\langle\rho, \alpha_{j}^{\vee}\right\rangle=1 \quad(1 \leq j \leq n) .
$$

1.2. For $\mu \in \mathbf{R}^{n}$, let $\Delta(\mu)=\sum_{w \in W} \operatorname{sgn}(w) e^{w \mu}$. There exist vectors $\mu_{1}, \ldots, \mu_{l} \in$ $\mathbf{R}^{n}$ and polynomials $F_{1}(u), \ldots, F_{l}(u)$ in the variables $u_{\alpha}, \alpha \in \Phi^{+}$, such that

$$
\sum_{w \in W} \operatorname{sgn}(w) w\left[e^{\rho} \prod_{\alpha>0} 1-u_{\alpha} e^{-\alpha}\right]=\sum_{i=1}^{l} F_{i}(u) \Delta\left(\mu_{i}\right) .
$$

Rearranging the term indexed by $w$, we obtain

$$
\begin{aligned}
e^{w \rho} \prod_{\alpha>0}\left(1-u_{\alpha} e^{-w \alpha}\right) & =\prod_{\alpha>0}\left(e^{w \alpha / 2}-u_{\alpha} e^{-w \alpha / 2}\right) \\
& =\operatorname{sgn}(w) \prod_{w \alpha<0}\left(u_{\alpha} e^{-w \alpha / 2}-e^{w \alpha / 2}\right) \prod_{w \alpha>0}\left(e^{w \alpha / 2}-u_{\alpha} e^{-w \alpha / 2}\right) \\
& =\operatorname{sgn}(w) e^{\rho} \prod_{w \alpha<0}\left(u_{\alpha}-e^{w \alpha}\right) \prod_{w \alpha>0}\left(1-u_{\alpha} e^{-w \alpha}\right),
\end{aligned}
$$

where each product is implicitly restricted to $\alpha>0$. Here we have used only the fact that $\operatorname{sgn}(w)=(-1)^{|\Phi(w)|}$, a corollary of the fact that $\ell(w)=|\Phi(w)|$ (e.g., $[\mathrm{H}$, $\S 1.7])$. Thus (2) can be rewritten in the form

$$
\sum_{w \in W} \prod_{w \alpha<0}\left(u_{\alpha}-e^{w \alpha}\right) \prod_{w \alpha>0}\left(1-u_{\alpha} e^{-w \alpha}\right)=e^{-\rho} \sum_{i=1}^{l} F_{i}(u) \Delta\left(\mu_{i}\right) .
$$

1.3. Define $\delta \in \mathbf{R}^{n}$ so that $\left\langle\alpha_{i}, \delta\right\rangle=1$ for $1 \leq i \leq n$. The height of $\mu \in \mathbf{R}^{n}$, denoted $\operatorname{ht}(\mu)$, is defined to be $\langle\mu, \delta\rangle$. Since $\Delta(w \mu)=\operatorname{sgn}(w) \Delta(\mu)$, we may insist that each $\mu_{i}$ appearing in (2) and (3) has maximum height among all members of its $W$-orbit. This requires $\left\langle\mu_{i}, \delta\right\rangle \geq\left\langle s_{j} \mu_{i}, \delta\right\rangle=\left\langle\mu_{i}, \delta\right\rangle-\left\langle\mu_{i}, \alpha_{j}^{\vee}\right\rangle$, or equivalently, $\left\langle\mu_{i}, \alpha_{j}^{\vee}\right\rangle \geq 0$ for $1 \leq j \leq n$. In fact, we may insist that

$$
\left\langle\mu_{i}, \alpha_{j}^{\vee}\right\rangle>0 \quad(1 \leq j \leq n)
$$

since equality would imply $s_{j} \mu_{i}=\mu_{i}$ and $\Delta\left(\mu_{i}\right)=0$.

Henceforth, we assume that $\Phi$ is crystallographic.

1.4. Every exponential that occurs when the left side of (3) is expanded is clearly of the form $e^{-\lambda}$, where $\lambda$ is a sum of positive roots. It follows that for each $i$ we must have $\mu_{i}=\rho-\lambda$ for some $\lambda$ of this form. Combining (1) and (4), we obtain that for such $\lambda$, we must have $\left\langle\lambda, \alpha_{j}^{\vee}\right\rangle<1$ for $1 \leq j \leq n$. Since $\Phi$ is assumed to be crystallographic, the quantities $\left\langle\lambda, \alpha_{j}^{\vee}\right\rangle$ are integers, and thus $\left\langle\lambda, \alpha_{j}^{\vee}\right\rangle \leq 0$. However, $\lambda$ is in the positive span of the positive roots (and hence also the co-roots), so 
$\langle\lambda, \lambda\rangle \leq 0$ and therefore $\lambda=0$. In other words, there is only one summand on the right side of $(2)$ and $\mu_{1}=\rho$, so

$$
\sum_{w \in W} \operatorname{sgn}(w) w\left[e^{\rho} \prod_{\alpha>0} 1-u_{\alpha} e^{-\alpha}\right]=F(u) \Delta(\rho),
$$

for some polynomial $F(u)$.

1.5. One may determine $F(u)$ by extracting the coefficient of $e^{\rho}$ in (2), or equivalently the constant term in (3). However, (3) is a polynomial in the variables $e^{-\alpha_{1}}, \ldots, e^{-\alpha_{n}}$, so the constant term can be obtained via the specialization $e^{-\alpha_{i}} \rightarrow$ 0, yielding

$$
F(u)=\sum_{w \in W} \prod_{\alpha \in \Phi(w)} u_{\alpha}
$$

1.6. Since the expression $e^{\rho} \prod_{\alpha>0}\left(1-e^{-\alpha}\right)=\prod_{\alpha>0}\left(e^{\alpha / 2}-e^{-\alpha / 2}\right)$ is antisymmetric with respect to the action of $W$, by setting $u_{\alpha}=1$ in (5) and (6) and cancelling a factor of $|W|$, we obtain the Weyl denominator formula

$$
\Delta(\rho)=e^{\rho} \prod_{\alpha>0}\left(1-e^{-\alpha}\right)=\prod_{\alpha>0}\left(e^{\alpha / 2}-e^{-\alpha / 2}\right) .
$$

Combining (5)-(7) yields

Theorem 1 (Macdonald). If $\Phi$ is crystallographic, then

$$
\sum_{w \in W} \operatorname{sgn}(w) e^{w \rho} \prod_{\alpha>0}\left(1-u_{\alpha} e^{-w \alpha}\right)=e^{\rho} \prod_{\alpha>0}\left(1-e^{-\alpha}\right) \cdot \sum_{w \in W} \prod_{\alpha \in \Phi(w)} u_{\alpha} .
$$

1.7. Following $[\mathrm{M}]$, we now specialize Theorem 1 by setting $u_{\alpha}=q$ and $e^{\mu}=$ $q^{-\langle\mu, \delta\rangle}$. For each $w \neq 1$, there must exist a simple root $\alpha_{i}$ such that $w^{-1} \alpha_{i}<0$ (e.g., $[\mathrm{H}, \S 1.8]$ ). It follows that for $\alpha=-w^{-1} \alpha_{i}$, we have $1-u_{\alpha} e^{-w \alpha}=0$, so the only nonzero term on the left side of (8) occurs when $w=1$. Hence Theorem 1 implies

$$
q^{-\langle\rho, \delta\rangle} \prod_{\alpha>0}\left(1-q^{\mathrm{ht}(\alpha)+1}\right)=q^{-\langle\rho, \delta\rangle} \prod_{\alpha>0}\left(1-q^{\mathrm{ht}(\alpha)}\right) \sum_{w \in W} q^{|\Phi(w)|} .
$$

Since $\ell(w)=|\Phi(w)|$, this proves

Corollary 2 (Macdonald). If $\Phi$ is crystallographic, then

$$
\sum_{w \in W} q^{\ell(w)}=\prod_{\alpha>0} \frac{1-q^{\mathrm{ht}(\alpha)+1}}{1-q^{\mathrm{ht}(\alpha)}} .
$$

\section{THE ORDERS OF REAL REFLECTION GROUPS}

Let us now discard the hypothesis that $\Phi$ is crystallographic. Although the formula for the Poincaré series given in Corollary 2 cannot be valid at this level of generality (it is not even a rational function of $q$ ), we claim that it $i s$ valid in the limit $q \rightarrow 1$; i.e.,

Theorem 3. If $W$ is a real reflection group, then

$$
|W|=\prod_{\alpha>0} \frac{\mathrm{ht}(\alpha)+1}{\mathrm{ht}(\alpha)} .
$$


For example, if $W$ is the dihedral group of order 10, then the coordinates of the positive roots with respect to the simple roots are $(1,0),(0,1),(\gamma, 1),(1, \gamma)$, and $(\gamma, \gamma)$, where $\gamma=(1+\sqrt{5}) / 2$ denotes the golden ratio. Thus (9) takes the form

$$
|W|=\frac{2 \cdot 2 \cdot(2+\gamma) \cdot(2+\gamma) \cdot(1+2 \gamma)}{1 \cdot 1 \cdot(1+\gamma) \cdot(1+\gamma) \cdot 2 \gamma} .
$$

In the following, it will be convenient to drop the pretense that $e^{\mu}$ denotes a "formal" exponential, and instead identify $e^{\mu}$ with the function $\nu \mapsto e^{\langle\mu, \nu\rangle}$ on $\mathbf{R}^{n}$. The group ring of $\mathbf{R}^{n}$ is now a function space, so let $\Delta(\mu)(\nu)$ denote the evaluation of $\Delta(\mu)$ at $\nu$.

Lemma 4. If $m=\left|\Phi^{+}\right|$, then for all $\mu, \nu \in \mathbf{R}^{n}, \lim _{q \rightarrow 1} \frac{\Delta(\mu)(\nu \log q)}{(1-q)^{m}}$ is finite.

Proof. Since $G(q)=\Delta(\mu)(\nu \log q)$ is analytic at $q=1$, it suffices to show that the Taylor series of $G$ vanishes through terms of order less than $m$. For this, consider that

$$
\frac{1}{i !} \frac{\partial^{i}}{\partial q^{i}} G(q)=\frac{1}{i !} \frac{\partial^{i}}{\partial q^{i}}\left[\sum_{w \in W} \operatorname{sgn}(w) q^{\langle w \mu, \nu\rangle}\right] \rightarrow \sum_{w \in W} \operatorname{sgn}(w)\left(\begin{array}{c}
\langle w \mu, \nu\rangle \\
i
\end{array}\right)
$$

in the limit $q \rightarrow 1$. However, as a function of $\nu$, the latter expression is a polynomial function of degree $\leq i$ that is antisymmetric with respect to the action of $W$. All such polynomials are divisible by the polynomial function $\nu \mapsto \prod_{\alpha>0}\langle\alpha, \nu\rangle$ (e.g., [H, 3.13]), and hence have degree at least $m$.

Proof of Theorem 3. Setting $u_{\alpha}=1$ in (2), we obtain

$$
|W| \cdot e^{\rho} \prod_{\alpha>0}\left(1-e^{-\alpha}\right)=\sum_{i=1}^{l} F_{i}(1) \Delta\left(\mu_{i}\right),
$$

by reasoning similar to 1.6. Evaluating this identity at $-\delta \log q$ yields

$$
|W|=q^{\langle\rho, \delta\rangle} \sum_{i=1}^{l} F_{i}(1) \frac{\Delta\left(\mu_{i}\right)(-\delta \log q)}{\Pi(q)},
$$

where $\Pi(q)=\prod_{\alpha>0}\left(1-q^{\mathrm{ht}(\alpha)}\right)$. In the limit $q \rightarrow 1$, we therefore have

$$
|W|=\sum_{i=1}^{l} c_{i} F_{i}(1),
$$

where $c_{i}=\lim _{q \rightarrow 1} \Delta\left(\mu_{i}\right)(-\delta \log q) / \Pi(q)$, a limit whose existence follows from Lemma 4. On the other hand, if we apply the reasoning of 1.7 to (2), we obtain

$$
q^{-\langle\rho, \delta\rangle} \prod_{\alpha>0}\left(1-q^{\mathrm{ht}(\alpha)+1}\right)=\sum_{i=1}^{l} F_{i}(q) \Delta\left(\mu_{i}\right)(-\delta \log q),
$$

or equivalently,

$$
\prod_{\alpha>0} \frac{1-q^{\mathrm{ht}(\alpha)+1}}{1-q^{\mathrm{ht}(\alpha)}}=q^{\langle\rho, \delta\rangle} \sum_{i=1}^{l} F_{i}(q) \frac{\Delta\left(\mu_{i}\right)(-\delta \log q)}{\Pi(q)} .
$$


Thus in the limit $q \rightarrow 1$, we obtain

$$
\prod_{\alpha>0} \frac{\operatorname{ht}(\alpha)+1}{\operatorname{ht}(\alpha)}=\sum_{i=1}^{l} c_{i} F_{i}(1)=|W| .
$$

\section{REFERENCES}

[Be] R. Beerends, "On the Abel Transform and its Inversion," Ph. D. thesis, University of Leiden, 1987.

[B] N. Bourbaki, "Groupes et Algèbres de Lie, Chaps. IV-VI," Masson, Paris, 1981. MR 83g:17001

[H] J. E. Humphreys, "Reflection Groups and Coxeter Groups," Cambridge Univ. Press, Cambridge, 1990. MR 92h:20002

[M] I. G. Macdonald, The Poincaré series of a Coxeter group, Math. Ann. 199 (1972), 161-174. MR 48:433

Department of Mathematics, University of Michigan, Ann Arbor, Michigan 481091109 